\title{
Ultra-high field (7T) functional magnetic resonance imaging in amyotrophic lateral sclerosis: a pilot study
}

Robert L. Barry ${ }^{1,2,3, \dagger, *}$, Suma Babu ${ }^{4,5, \dagger, *}$, Sheeba Arnold Anteraper ${ }^{6,7,8}$, Christina Triantafyllou ${ }^{1,2,9}$, Boris Keil ${ }^{1,2,10}$, Olivia E. Rowe ${ }^{1}$, D. Rangaprakash ${ }^{1,2}$, Sabrina Paganoni ${ }^{4,11,12}$, Robert Lawson ${ }^{4}$, Christina Dheel ${ }^{4}$, Paul M. Cernasov ${ }^{4}$,

Bruce R. Rosen ${ }^{1,2,3}$, Eva-Maria Ratai ${ }^{1,13,2, \ddagger}$, and Nazem Atassi ${ }^{4,5,14, \ddagger}$

${ }^{1}$ Athinoula A. Martinos Center for Biomedical Imaging, Department of Radiology, Massachusetts General Hospital, Charlestown, MA, USA

${ }^{2}$ Department of Radiology, Harvard Medical School, Boston, MA, USA

${ }^{3}$ Harvard-Massachusetts Institute of Technology Health Sciences \& Technology, Cambridge, MA, USA

${ }^{4}$ Sean M. Healey \& AMG Center for ALS at Massachusetts General Hospital, Department of Neurology, Neurological Clinical Research Institute, Boston, MA, USA

${ }^{5}$ Department of Neurology, Harvard Medical School, Boston, MA, USA

${ }^{6}$ Department of Psychology, Northeastern University, Boston, MA, USA

${ }^{7}$ Department of Psychiatry, Massachusetts General Hospital, Boston, MA, USA

${ }^{8}$ Department of Brain and Cognitive Sciences, Massachusetts Institute of Technology, Cambridge, MA, USA

${ }^{9}$ Siemens Healthineers, Erlangen, Germany

${ }^{10}$ Mittelhessen University of Applied Sciences, Department of Life Science Engineering, Institute of Medical Physics and Radiation Protection, Giessen, Germany

${ }^{11}$ Spaulding Rehabilitation Hospital, Charlestown, MA, USA

${ }^{12}$ Department of Physical Medicine and Rehabilitation, Harvard Medical School, Boston, MA, USA

${ }^{13}$ Division of Neuroradiology, Massachusetts General Hospital, Boston, MA, USA

${ }^{14}$ Sanofi Genzyme, Cambridge, MA, USA

${ }^{\dagger}$ These authors contributed equally to this work.

$\ddagger$ These authors share senior authorship.

*Corresponding authors (Robert.Barry@mgh.Harvard.edu and/or sbabu@mgh.Harvard.edu)

Compiled by LTEX on: 02/19/2021 at 16:36:30 


\begin{abstract}
Amyotrophic lateral sclerosis (ALS) is a neurodegenerative disease of the central nervous system that results in a progressive loss of motor function and ultimately death. It is critical, yet also challenging, to develop non-invasive biomarkers to identify, localize, measure and/or track biological mechanisms implicated in ALS. Such biomarkers may also provide clues to identify potential molecular targets for future therapeutic trials. Herein we report on a pilot study involving twelve participants with ALS and nine age-matched healthy controls who underwent high-resolution resting state functional magnetic resonance imaging at an ultra-high field of 7 Tesla. A group-level whole-brain analysis revealed a disruption in longrange functional connectivity between the superior sensorimotor cortex (in the precentral gyrus) and bilateral cerebellar lobule VI. Post hoc analyses using atlas-derived left and right cerebellar lobule VI revealed decreased functional connectivity in ALS participants that predominantly mapped to bilateral postcentral and precentral gyri. Cerebellar lobule VI is a transition zone between anterior motor networks and posterior non-motor networks in the cerebellum, and is associated with a wide range of key functions including complex motor and cognitive processing tasks. Our observation of the involvement of cerebellar lobule VI adds to the growing number of studies implicating the cerebellum in ALS. Future avenues of scientific investigation should consider how high-resolution imaging at $7 \mathrm{~T}$ may be leveraged to visualize differences in functional connectivity disturbances in various genotypes and phenotypes of ALS along the ALS-frontotemporal dementia spectrum.
\end{abstract}

\title{
1 Introduction
}

Amyotrophic lateral sclerosis (ALS) is characterized by the progressive degeneration of motor neurons and their axonal connections in the brain and spinal cord. The disease presents as a continuous loss of motor functions and ultimately results in death. ALS is known to be associated in many individuals with a spectrum of extra-motor dysfunction including behavioral changes, cognitive impairment, dementia and mood changes ${ }^{1}$. Although rare, there are growing observations that some individuals with "ALS plus" syndromes may have other associated neurological features including extrapyramidal/parkinsonian features, diminished taste/smell, cerebellar ataxia, parasomnias, or eye gaze abnormalities ${ }^{1-8}$. This suggests that ALS may also be viewed as an overlapping neurodegenerative disorder with widespread involvement of different brain regions.

From a neuropathological standpoint, ALS is recognized as a TDP43 proteinopathy in $98 \%$ of all cases (except forms of ALS with SOD1 and FUS gene expressions) ${ }^{9}$. The Braak staging of spreading TDP43 inclusion pathology burden in a large postmortem ALS cohort suggests that the disease pathology extends beyond the well known motor cortical system in later stages of disease to also include precerebellar nuclei, prefrontal cortex, and the striatum ${ }^{10}$. There is an unmet need to expand ongoing in vivo clinical research to evaluate reliable and sensitive neuroimaging biomarkers ${ }^{11}$ that can identify and map neural networks 
that degenerate with ALS and more importantly their relevance and predictability to clinical disability progression ${ }^{12-20}$. There has also been growth in multimodal neuroimaging techniques in the past decade, which provide unique opportunities to leap forward toward developing novel and quantitative biomarkers of disease localization, tracking disease progression, and improving phenotypic classifications in ALS ${ }^{21-23}$.

At 1.5 or 3 Tesla, diffusion magnetic resonance imaging (MRI) studies of ALS have revealed widespread structural damage across motor and non-motor regions ${ }^{24-38}$. The role of functional MRI (fMRI) in the study of neurodegenerative disorders continues to expand ${ }^{39}$ and maps brain networks based upon the temporal coherency of hemodynamic responses in a 'resting state' or evoked via one or more tasks. Prior fMRI studies at 1.5 or $3 \mathrm{~T}$ (and one at 4.7T) have revealed the impact of disease on brain functions ${ }^{40-45,26,28,46-53}$ and

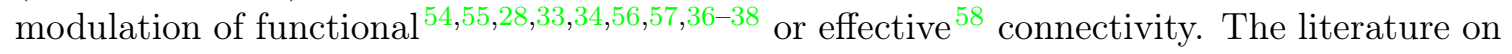
resting state fMRI (rs-fMRI) in ALS is relatively sparse, and there is minimal convergence of findings across different cross-sectional and longitudinal cohorts ${ }^{59}$. However, several rsfMRI studies have noted aberrations (increases or decreases) in functional connectivity in ALS participants involving the sensorimotor network (SMN) ${ }^{54,28,56,33,36,38}$ as well as other intrinsic networks ${ }^{60}$.

To date, only a handful of 7 Tesla (7T) ALS studies have been published in the brain ${ }^{61-67}$ and spinal cord ${ }^{68}$. The benefits of imaging at an ultra-high field of $7 \mathrm{~T}$ include increases in spatial signal-to-noise and contrast-to-noise ratios, functional contrast-to-noise ratio, and spectral dispersion ${ }^{69-72}$. In the cervical cord, high-resolution $\mathrm{T}_{2}{ }^{*}$-weighted images demonstrated sharp delineation between gray and white matter, and white matter $\mathrm{T}_{2}{ }^{*}$ hyperintensities were visualized in regions of ALS pathology ${ }^{68}$. In the brain, a previous $7 \mathrm{~T}$ ALS study reported hyperintensities in $\mathrm{R}_{2} *$ maps in the motor cortex of ALS participants, which correlated with iron accumulation in post mortem pathological studies ${ }^{62}$. Anatomical imaging of the deep layers of the primary motor cortex at 7T revealed atrophy and $\mathrm{T}_{2}{ }^{*}$ hypointensities in participants with ALS that correlated with upper motor neuron (UMN) impairment and disease progression rate ${ }^{63}$, and increases in magnetic susceptibility measured via quantitative susceptibility mapping co-localized with $\mathrm{T}_{2}{ }^{*}$ hypointensities in the middle and deep layers ${ }^{64}$. Diffusion imaging at $7 \mathrm{~T}$ did not reveal whole-brain differences between controls and ALS participants, but analyses of the corticospinal tracts (CSTs) revealed decreased fractional anisotrophy and increased magnetization transfer ratio in ALS participants ${ }^{65}$. Single voxel magnetic resonance spectroscopy (MRS) of the left precentral gyrus at $7 \mathrm{~T}$ revealed decreases in $N$-acetylaspartate (NAA), glutamate, and total NAA ( $N$-acetylaspartate $+N$-acetylaspartylglutamate) in ALS participants, which correlated with forced vital capacity; additionally, the ratio of total NAA to total creatine (creatine+phosphocreatine) correlated with overall functional decline as measured by decreasing ALS Functional Rating Scale-Revised (ALSFRS-R) ${ }^{66}$ scores. Another 7T MRS study reported a lower ratio of total NAA to myo-inositol in both the motor cortex and pons of ALS participants, and that levels of total NAA, myo-inositol, and glutamate in the motor cortex of ALS participants were influenced by the extent of disease progression ${ }^{67}$. 
We are not aware of previous reports studying ALS using 7T fMRI. Therefore, the pilot study presented herein contributes to the existing body of $7 \mathrm{~T}$ work by investigating group-level differences in functional networks between healthy controls and ALS participants using high-resolution rs-fMRI with spatial coverage extending from the cerebrum to the cerebellum. We additionally assessed the association between significant imaging findings and ALS clinical variables.

\section{Material and methods}

\subsection{Study cohort}

This cross-sectional pilot study was conducted at the Athinoula A. Martinos Center for Biomedical Imaging, Massachusetts General Hospital, Charlestown, Massachusetts, USA. Scans were performed within a 4-year period between 2011 and 2015. Subjects included in the current study $(N=21)$ were drawn from a cohort of 25 subjects previously reported by our group ${ }^{66}$ who provided a high-resolution rs-fMRI scan upon completion of the main MRS protocol. All subjects provided written, informed consent through a protocol approved by the Partners Human Research Committee.

Twelve participants who met the revised El Escorial criteria for at least possible ALS and nine age-matched healthy controls were enrolled in this study. All ALS participants met the institutional MRI safety criteria and were able to tolerate lying flat for the scan duration. All participants had to have no other neurodegenerative disease diagnosis. Baseline characteristics of the study cohorts are detailed in Table 1. Family history of ALS was not collected for these participants. One ALS participant was confirmed to have C9orf72 repeat expansion. ALS causative genetic mutations remained unknown for the remaining 11 participants. All ALS participants underwent standard clinical outcome assessments collected by certified raters at a matching time point to the scan including ALSFRS-R ${ }^{73}$, slow vital capacity (SVC), and a quantitative muscle strength test using hand-held dynamometry.

\subsection{Data acquisition}

Experiments were performed on a whole-body 7 Tesla system (Siemens Healthineers, Erlangen, Germany) using a single-channel transmit birdcage volume coil and a custom-built 32-channel receive $\mathrm{coil}^{74}$.

All subjects completed a 45-min brain imaging session without contrast, which included a high-resolution T1-weighted anatomical scan, MRS (previously reported ${ }^{66}$ ), and a highresolution rs-fMRI scan. High-resolution anatomical images were acquired with the following parameters: field of view $(\mathrm{FOV})=256 \mathrm{~mm} \times 256 \mathrm{~mm} \times 176 \mathrm{~mm}$, voxel size $=1 \times 1 \times 1$ $\mathrm{mm}^{3}, \mathrm{TE}=1.48 \mathrm{~ms}$, inversion time $=1100 \mathrm{~ms}$, repetition time $(\mathrm{TR})=2530 \mathrm{~ms}$, flip angle $=7^{\circ}$. High-resolution blood oxygenation level dependent (BOLD) functional images were acquired with the following parameters: $\mathrm{FOV}=190 \mathrm{~mm} \times 190 \mathrm{~mm} \times 108 \mathrm{~mm}$, voxel size 
Table 1: Baseline characteristics for healthy controls and participants with ALS.

\begin{tabular}{llc}
\hline Baseline characteristic & $\begin{array}{l}\text { ALS }(n=12) \\
\%(n) / \mu(\sigma)\end{array}$ & $\begin{array}{c}\text { Controls }(n=9) \\
\%(n) / \mu(\sigma)\end{array}$ \\
\hline Age at screening (yrs) & $56.8(10.3)$ & $53.2(11.1)$ \\
Genetic abnormality & unknown $(11)$ & $\mathrm{n} / \mathrm{a}$ \\
& C9orf72 positive $(1)$ & \\
Male & $75 \%(9)$ & $55 \%(5)$ \\
Caucasian & $100 \%(12)$ & $100 \%(9)$ \\
Symptom onset to diagnosis (months) & $10.3(7.0)$ & $\mathrm{n} / \mathrm{a}$ \\
Symptom onset to scan (months) & $30.3(23.4)$ & $\mathrm{n} / \mathrm{a}$ \\
Limb onset & $67 \%(8)$ & $\mathrm{n} / \mathrm{a}$ \\
Baseline SVC (\% predicted) & $93.4 \%(22.2 \%)$ & $\mathrm{n} / \mathrm{a}$ \\
ALSFRS-R at baseline & $38.1(4.8)$ & $\mathrm{n} / \mathrm{a}$ \\
Estimated ALSFRS-R slope & $0.46(0.35)$ & $\mathrm{n} / \mathrm{a}$ \\
pre-baseline ((48 - baseline & & \\
ALSFRS-R) / disease duration) & & $\mathrm{n} / \mathrm{a}$ \\
(points/month) & & \\
Revised El Escorial criteria & definite (5) & \\
& probable (3) & \\
& probable lab supported (1) & \\
Riluzole use & possible (3) & \\
\hline
\end{tabular}

$=1.15 \times 1.15 \times 1.18 \mathrm{~mm}^{3}, \mathrm{TE}=22.6 \mathrm{~ms}, \mathrm{TR}=5000 \mathrm{~ms}$ (except one subject where $\mathrm{TR}=$ $5560 \mathrm{~ms}$ inadvertently), flip angle $=84^{\circ}$, number of volumes $=94$ (scan time $\left.=7.8 \mathrm{~min}\right)$. Subjects were instructed to remain still and in a state of wakeful rest prior to the functional scan. Reported fMRI parameters are the mean across subjects due to minor adjustments to the protocol over the course of the 4-year study, and subject-specific adjustments to obviate specific absorption rate limits and peripheral nerve stimulation.

\subsection{Data processing and analysis}

Data were processed and analyzed using the CONN toolbox ${ }^{75}$ release 18.b. Structural scans were translated to $(0,0,0)$ coordinates and normalized to Montreal Neurological Institute (MNI) space ${ }^{76}$ with simultaneous segmentations of gray matter, white matter, and cerebrospinal fluid (CSF). Functional scans were preprocessed using CONN's recommended pipeline for volume-based analyses: i) realignment and unwarping (including rigid-body motion estimation and correction), ii) centering to $(0,0,0)$ coordinates, iii) slice-timing correction, iv) ARtifact detection Tools (ART)-based outlier detection for data 'scrubbing' (www.nitrc.org/projects/artifact_detect) with thresholds of $\pm 3 \sigma$ for deviation from 
global mean and $1.5 \mathrm{~mm}$ for framewise displacement, v) normalization to MNI space with segmentations of tissues and CSF, and vi) spatial smoothing using an 8-mm full-widthat-half-maximum Gaussian kernel $^{77}$ to reduce between-subject anatomical variability in preparation for group analyses. Physiological noise regressors included five principal components of anatomically-derived white matter and CSF segments' time series ${ }^{78}$, respectively, the six estimated rigid-body motion parameters and their first derivatives, and ART-derived outliers. Additional preprocessing steps included linear detrending and band-pass filtering (0.008-0.09 Hz).

An exploratory second-level analysis of functional connectivity was performed using CONN's seed-to-voxel analysis tool that considers numerous seed regions throughout the brain. Each seed was a $2 \times 2 \times 2 \mathrm{~mm}^{3}$ region centered at a given MNI coordinate. A functional contrast of Controls $>$ ALS participants was selected to probe group-level differences between controls and participants with ALS. Results from this contrast (presented hereafter) were saved and projected onto a spatially unbiased atlas of the cerebellum using the spatially unbiased infra-tentorial template (SUIT) version 3.0 ${ }^{79-82}$. Post hoc seed-to-voxel analyses with the same contrast were then performed using three atlas (AAL)-derived regions: left cerebellar lobule VI, right cerebellar lobule VI, and combined (left and right) cerebellar lobule VI.

\section{Results}

The cohorts of ALS participants $(n=12)$ and controls $(n=9)$ did not differ significantly in age or $\operatorname{sex}(p=0.39$ and $p=0.38$, respectively, using Wilcoxon rank sum tests). The aggregate rigid-body motion parameters were also not significantly different between groups $(p=0.66, t$-test), which is an important quality assurance observation to ensure that group-level differences in functional connectivity were not erroneously driven by groupdependent motion ${ }^{83}$. The number of data points 'scrubbed' between groups was also not significantly different (median \pm median absolute deviation: $5 \pm 3.85$ for controls vs. $5 \pm 6.61$ for ALS participants; $p=0.83$ using a Wilcoxon rank sum test). No subjects were excluded due to excessive motion.

Across all regions considered in this exploratory analysis (voxel threshold: $p<0.005$ uncorrected, two-sided; cluster threshold: $p<0.05$ with false discovery rate correction), we observed a decrease in functional connectivity between the superior sensorimotor cortex (MNI coordinates $=(0,-31,67)$, which anatomically localizes to the inter-hemispheric fissure and in close proximity to the proximal leg representation area of the motor cortex) and lobule VI of the cerebellum in ALS participants vs. matched controls. Figure 1a presents the functional contrast Controls $>$ ALS participants overlaid onto a single 2D axial slice through the mid-cerebellum. While a bilateral region of the cerebellum exhibited higher connectivity in controls, no regions exhibited higher connectivity in ALS participants at the same threshold. (Additional post hoc analyses within groups showed that these results 
were due to an absence of significant correlations in ALS participants.) Figure 1b projects these results onto a cerebellar flatmap ${ }^{82}$. The group-level results are remarkably symmetric and predominantly mapped onto bilateral cerebellar lobule VI (involving both medial and lateral aspects of this cerebellar lobule).

Figure 2 visualizes results from three post hoc analyses using cerebellar lobule VI seed regions, presented as superior views of the partially-inflated cortical surface (voxel threshold: $p<0.001$ uncorrected, two-sided; cluster threshold: $p<0.05$ with false discovery rate correction). Group-level differences between ALS participants and controls predominantly mapped to the postcentral and precentral gyri with secondary differences in the superior parietal lobules and the precuneus cortex. Detailed results are presented in Table S1.

Additional analyses were performed correlating functional connectivity and ALS clinical metrics. These methods and results are presented in Supplementary Information. A meta-analysis of 57 ALS studies performed using NeuroQuery ${ }^{84}$, also presented in Supplementary Information, revealed activations in regions we found to be connected (i.e., somatosensory/motor cortex and cerebellum). 

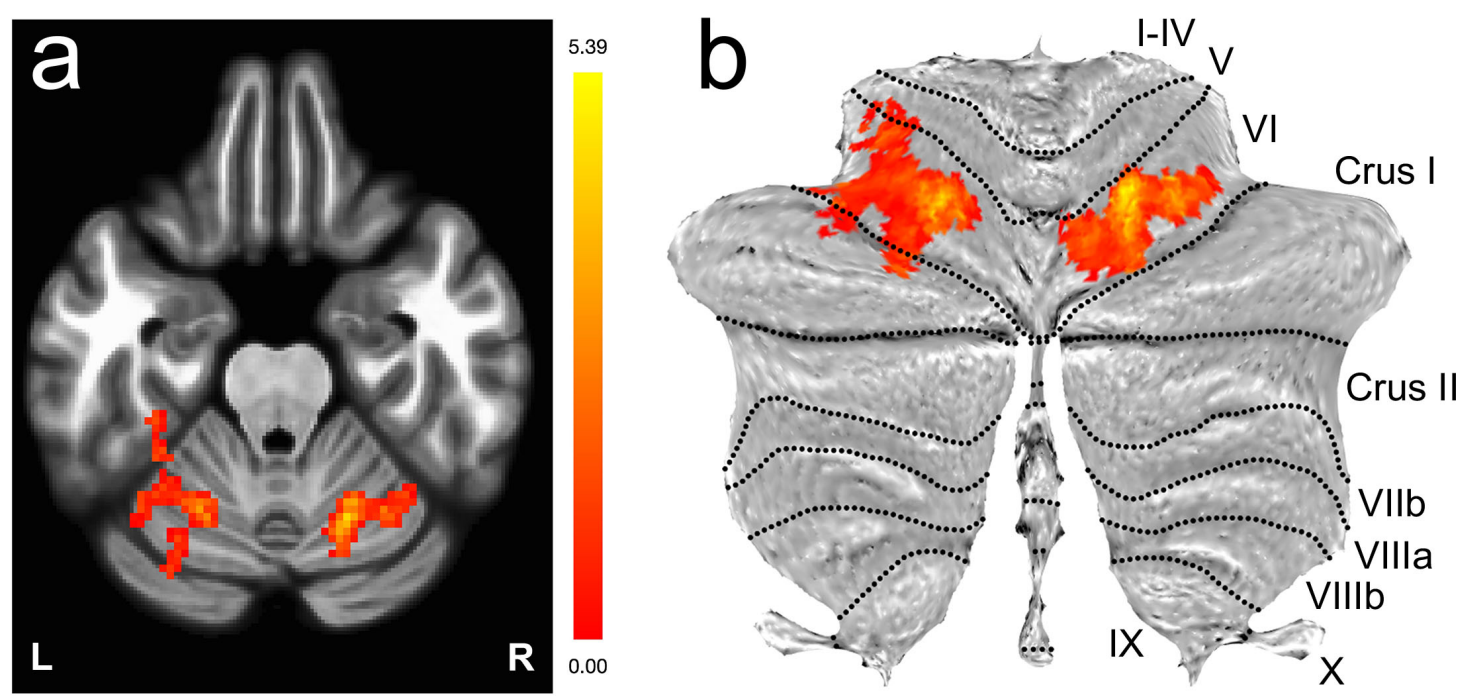

Figure 1: Decreased functional connectivity between the sensorimotor cortex and bilateral lobule VI of the cerebellum visualized via the functional contrast Controls $>$ ALS participants $(p<0.005$ uncorrected, two-sided; cluster threshold: $p<0.05$ with false discovery rate correction) for a seed region located in the superior sensorimotor cortex $(\mathrm{MNI}$ coordinates $=(0,-31,67))$. The results are presented as a) an overlay onto a single 2D axial slice through the cerebellum, and b) a projection onto a cerebellar flatmap ${ }^{82}$.
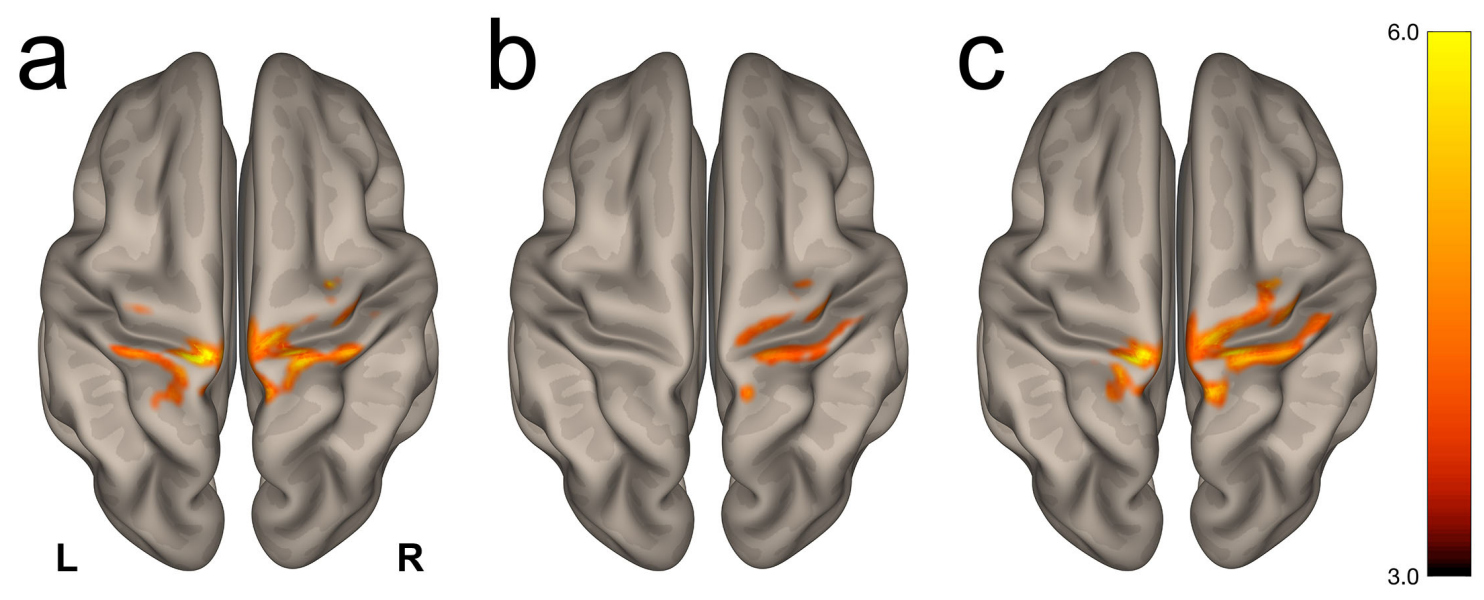

Figure 2: Visualization of the post hoc seed-to-voxel contrast Controls $>$ ALS participants using atlas-derived a) left, b) right, and c) bilateral cerebellar lobule VI as seed regions. Group-level differences $(p<0.001$ uncorrected, two-sided; cluster threshold: $p<0.05$ with false discovery rate correction) were predominantly observed in the postcentral and precentral gyri. The complete list of clusters is presented in Table S1. 


\section{Discussion}

In this data-driven, cross-sectional, ultra-high field rs-fMRI pilot study, we report significantly decreased functional connectivity between the superior sensorimotor cortex and bilateral cerebellar lobule VI in participants with ALS. Post hoc seed-to-voxel analyses of cerebellar lobule VI localize group-level differences in functional connectivity to bilateral precentral and postcentral gyri. While previous rs-fMRI studies have implicated the motor cortex and/or cerebellum in ALS, we are not aware of a previous report highlighting disruption in the specific link between the superior sensorimotor cortex and cerebellar lobule VI.

The upper motor neurons residing in the precentral gyri are well known to be involved in various disease mechanisms and neurodegeneration in $\mathrm{ALS}^{17}$. There is also growing evidence of the involvement of cerebellar structures in imaging and neuropathological studies in sporadic ALS, ALS/neurodegenerative syndromes arising due to C9orf72 or ataxin-2 repeat expansions and TBK1 mutations, and in primary lateral sclerosis (PLS) ${ }^{85-88}$. For example, pathological features associated with the production of C9orf72 transcripts with expanded repeats are the formation of nuclear ribonucleic acid foci in the frontal cortex, hippocampus, and cerebellum ${ }^{85}$. Functional and diffusion neuroimaging studies support topographical mappings of cerebellar lobule VI to premotor and primary motor cortices reciprocally ${ }^{89}$. Task-based fMRI has revealed that ocular and orofacial movements engage the medial aspect of cerebellar lobule $\mathrm{VI}^{90-94}$. During more complex motor tasks involving motor planning/learning or cognitive processing, activation patterns shift more laterally in lobules VI and VII ${ }^{95,96}$. Previous rs-fMRI studies have reported on such cortical-cerebellar circuits in healthy volunteers ${ }^{97-99}$, further supporting the feasibility that this network is susceptible to disease-related impairment. The multifaceted role of cerebellar lobule VI therefore underscores the significance of its involvement in degeneration in ALS.

Previous studies have reported that cerebellar lobule VI and other regions of the cerebellum play a key role as a hub for diverse and integral motor and non-motor functions in ALS. A recent 3T study using an event-related motor paradigm reported the involvement of lobule VI in ALS participants with UMN predominant dysfunction ${ }^{100}$. A 3T diffusion tensor imaging (DTI) analysis of white matter tracts in ALS and PLS participants with pseudobulbar affect found abnormalities in the frontotemporal cortex, transverse pontine, and middle cerebellar peduncular circuits ${ }^{101}$. A recent longitudinal 3T voxel-based morphometry study in sporadic ALS (excluding C9orf72 ALS participants) found significant cerebellar gray matter density reductions in multiple regions including bilateral cerebellar lobule VI that evolved on subsequent follow-up scans compared to matched controls ${ }^{102}$. Another 3T DTI study showed widespread disruption of cerebellar white matter tracts along the dentato-rubro-thalamo-cortical and spino-cerebellar pathways in PLS that may be greater than ALS, implying the impact of longer duration motor neuron disease ${ }^{103}$. Such studies highlight the fact that studying the cerebellum and its neural connections is an important endeavor - yet remains challenging due to the relatively small size of many 
key anatomical structures.

The functional involvement of the cerebellum in $\mathrm{ALS}^{104}$ has been reported in taskbased $^{41,42,105,100}$ and resting state $28,106,33,38$ fMRI studies at lower fields, and functional changes have been correlated to disease severity ${ }^{106}$, clinical variables ${ }^{100}$, and task difficulty $^{41}$. However, participants with ALS have a reduced capacity for performing motor tasks associated with task-based fMRI, so rs-fMRI ${ }^{54,55,33,34,58,57,37,38}$, along with motor imagery paradigms ${ }^{45}$, are attractive options in studying diseases with motor implications. There is considerable variability in the literature on functional connectivity network patterns in ALS. Beyond motor task-related disability and heterogeneity in ALS participant characteristics, there are of course other challenges for developing and scaling fMRI as a biomarker for tracking disease progression in ALS including technological (e.g., scanner vendor, field strength) and methodological/statistical (acquisition, preprocessing choices, hypothesis- vs. data-driven approaches) considerations ${ }^{107,59}$.

Earlier studies have reported abnormal connectivity/function involving the cerebellar and SMN areas in ALS ${ }^{28,41,38}$. Specifically, 3T studies have highlighted the implications of ALS upon cerebello-cortical pathways. Both ALS participants and ALS mutation carriers have exhibited increased functional connectivity between the cerebellum and the precuneuscingulate-middle frontal resting state network compared to controls, which may indicate pre-symptomatic ALS cerebral pathology ${ }^{33}$. ALS participants have also shown decreased functional connectivity between the dentate nucleus in the cerebellum and both the supplementary motor area and left cerebellum lobule IV, which correlated positively with ALSFRS-R ${ }^{38}$. These alterations in functional connectivity, especially regarding cerebellar lobule IV and the primary motor cortex, may indicate alterations in the spino-cerebellar tract $^{38}$. Furthermore, SMN correlations may increase in the earlier stage of disease ${ }^{28}$ and then decrease with more severe and longer duration disease ${ }^{56,36}$. An early $1.5 \mathrm{~T}$ study reported decreased functional connectivity between the right SMN and right cerebellar lobule VI in ALS participants vs. healthy controls, but only in a subset of 16 ALS participants with CST damage (as measured via decreased fractional anisotrophy relative to controls and also ALS participants with undetectable CST damage) and only within the right hemisphere $^{28}$. A longitudinal rs-fMRI study (with 13 ALS and 3 PLS participants) observed functional connectivity decreases between the SMN and frontal pole and increases between the left fronto-parietal network and left primary motor cortex ${ }^{36}$. Another longitudinal 3T study (27 ALS participants vs. 56 controls) reported expansions in intrinsic motor, brainstem, ventral attention, and default mode/hippocampal networks that appeared to follow the same neuropathological pattern seen in Braak's TDP43 staging in ALS ${ }^{34}$. However, a cross-sectional study at 4.7T (20 ALS participants vs. 34 controls) used 10-mm diameter spheres within the pre- and post-central gyri and supplemental motor area to form a combined SMN time course and reported no significant group-level differences in the default mode network or SMN, or between subgroups of high and low UMN burden participants ${ }^{56}$.

Due to higher signal-to-noise and functional contrast-to-noise ratios, unique spatial contrasts, and increasing availability of $7 \mathrm{~T}$ systems, high-resolution structural and functional 
imaging at $7 \mathrm{~T}$ is poised to provide new insights into disease manifestation and progres$\operatorname{sion}^{108}$ in ALS as well as other diseases. The primary advantages of $7 \mathrm{~T}$ fMRI over lower fields is the greater-than-linear increase in BOLD sensitivity ${ }^{109,110}$ that permits smaller voxels without losing statistical power, and weighting toward smaller vessels supplying the superficial cortical structures, such as sensorimotor cortices, and smaller infratentorial tissue structures in close proximity to air-fluid-tissue interfaces such as cerebellum. One must keep in mind, however, that the mechanisms that give rise to an increase in BOLD contrast at higher fields also increase physiological noise ${ }^{111}$, and thus care must be taken to mitigate confounding noise sources due to respiration, cardiac pulsatility, and subject motion. In this $7 \mathrm{~T}$ study, the influence of physiological noise was modeled in CONN using ARTderived outliers, five principal components of anatomically-derived white matter and CSF segments, and the six estimated rigid-body motion parameters and their first derivatives. The appropriate impact of these regressors on each subject's data was then reviewed using CONN's suite of tools to visualize first-level fMRI data before and after denoising.

Our investigation has limitations that must, of course, be mentioned. First, this pilot study is a cross-sectional design with relatively small group sizes, so we are therefore not characterizing the trajectory of disease progression for individual participants but rather are performing a group-level comparison that includes inherent heterogeneity within the cohort of ALS participants. Second, while there is one identified C9orf72 positive ALS participant in this cohort, the genetic status and hence contribution of other non-genotyped ALS participants to the abnormal cerebellar connectivity results is unknown. Third, the fMRI data were acquired with a longer TR than what is commonly used nowadays, resulting in fewer degrees of freedom and limitations on how the analyses may be conducted. It is important to note that the high-resolution acquisition strategy was implemented with the methods available when this study commenced in mid-2011 to obtain fMRI data in a regime expected to have minimal physiological noise ${ }^{112,113}$. Single-subject analyses were initially pursued in the analyses of these data, but the resultant degrees of freedom per subject after denoising necessitated the second-level analyses presented herein. A longer TR additionally precludes the study of higher frequency resting state signals - up to $0.8 \mathrm{~Hz}$ - which has become an important area of investigation in recent years ${ }^{114-117}$. Thus, future studies should use a TR of 1-1.5 sec and also acquire a longer resting state run to increase the fidelity of functional connectivity estimates ${ }^{118}$. Finally, due to the high-resolution wholebrain acquisition strategy, the imaging FOV did not include the more caudal regions of the cerebellum; therefore, the results presented in this study cannot preclude the possible involvement of these regions of the cerebellum.

Reports of modulation in the cerebellum support the evidence that there are not only adaptive intracortical changes ${ }^{119,40,41,45,55,48,106,51,37,100}$ in response to neurodegeneration in ALS, but also functional reorganization that occurs subcortically $42,43,106,51,100$. In sum, these reports highlight the brain's widespread plasticity and capacity to partially overcome cortical and spinal neuronal degeneration. (Our post hoc analysis of functional modulation restricted to the subcortex ${ }^{120}$ may be found in Supplementary Information.) Future 
studies in ALS may employ recent acquisition advances, most notably simultaneous multislice imaging ${ }^{121}$, to acquire high-resolution $7 \mathrm{~T}$ fMRI data from the cerebrum and entire cerebellum with a reduced volume acquisition time. Accurate measurements of cerebellar volume should also be obtained to investigate possible relationships between changes in BOLD signals and gray matter atrophy. In addition to ALSFRS-R, the collection of neurocognitive clinical measures should be considered ${ }^{122}$. Ultimately, larger cohorts and longitudinal studies at ultra-high field are necessary in various genetic subgroups and phenotypes of UMN-predominant motor neuron diseases to better understand the complex functional connectivity architecture from standpoints of both clinical relevance and tracking disease progression. Results from such studies may have a broader impact to inform the design of fMRI studies to better understand the involvement of various neural systems in other neurodegenerative diseases - including Alzheimer's disease, Parkinson's disease, Huntington's disease, multiple sclerosis, frontotemporal dementia, and PLS - where the cerebellum is thought to be affected under the shadows of other key motor and cognitive neural pathways ${ }^{123,124,103}$.

\section{Conclusions}

This pilot study acquired 7 Tesla resting state fMRI data in healthy controls and participants with ALS. An exploratory whole-brain analysis revealed a disruption in functional connectivity between the superior sensorimotor cortex (in the precentral gyrus) and bilateral cerebellar lobule VI. Post hoc analyses using atlas-derived left and right cerebellar lobule VI revealed decreased functional connectivity in ALS participants that predominantly mapped to bilateral postcentral and precentral gyri. Cerebellar lobule VI is associated with a wide range of key functions including complex motor and cognitive processing tasks. These findings add to the growing number of ALS reports implicating the cerebellum, and future studies are required with larger cohorts and known genetic status for all ALS participants.

\section{Acknowledgments}

The authors acknowledge the generosity of our participants and their families. We also thank Dr. Alfonso Nieto-Castañón for helpful discussions on data preprocessing using the CONN toolbox. Imaging was performed at the Athinoula A. Martinos Center for Biomedical Imaging at the Massachusetts General Hospital using resources provided by the Center for Functional Neuroimaging Technologies (P41EB015896) and the Center for Mesoscale Mapping (P41EB030006), Biotechnology Resource Grants supported by the National Institute of Biomedical Imaging and Bioengineering, National Institutes of Health (NIH). The NIH also provided support through grants R00EB016689 and R01EB027779 (R.L.B.) and K23NS083715 (N.A). This research was also supported in part by the Harvard NeuroDiscovery Center, the Muscular Dystrophy Association, and the American Academy of 
Neurology (N.A.), and by the MGH/HST Athinoula A. Martinos Center for Biomedical Imaging. The content is solely the responsibility of the authors and does not necessarily represent the official views of the NIH.

\section{Competing interests}

Christina Triantafyllou, PhD, is currently employed by Siemens Healthineers. Nazem Atassi, MD, PhD, is currently employed by Sanofi Genzyme. All other authors declare no competing interests related to this work.

\section{References}

[1] Turner MR, Goldacre R, Talbot K, Goldacre MJ. Psychiatric disorders prior to amyotrophic lateral sclerosis. Ann Neurol 2016;80:935-938.

[2] Brooks BR. ALS-Plus - where does it begin, where does it end? J Neurol Sci $2014 ; 345: 1-2$.

[3] Al-Chalabi A, Hardiman O, Kiernan MC, Chiò A, Rix-Brooks B, van den Berg LH. Amyotrophic lateral sclerosis: moving towards a new classification system. Lancet Neurol 2016;15:1182-1194.

[4] Abrahams S, Goldstein LH, Kew JJ, Brooks DJ, Lloyd CM, Frith CD, et al. Frontal lobe dysfunction in amyotrophic lateral sclerosis. A PET study. Brain 1996;119 Pt 6:2105-2120.

[5] Abrahams S, Leigh PN, Goldstein LH. Cognitive change in ALS: a prospective study. Neurology 2005;64:1222-1226.

[6] Lulé D, Diekmann V, Anders S, Kassubek J, Kübler A, Ludolph AC, et al. Brain responses to emotional stimuli in patients with amyotrophic lateral sclerosis (ALS). J Neurol 2007;254:519-527.

[7] Silani V, Ludolph A, Fornai F. The emerging picture of ALS: a multisystem, not only a "motor neuron disease". Arch Ital Biol 2017;155:153-158.

[8] Bede P, Omer T, Finegan E, Chipika RH, Iyer PM, Doherty MA, et al. Connectivitybased characterisation of subcortical grey matter pathology in frontotemporal dementia and ALS: a multimodal neuroimaging study. Brain Imaging Behav 2018;12:16961707 .

[9] Feneberg E, Gray E, Ansorge O, Talbot K, Turner MR. Towards a TDP-43-based biomarker for ALS and FTLD. Mol Neurobiol 2018;55:7789-7801. 
[10] Brettschneider J, Del Tredici K, Toledo JB, Robinson JL, Irwin DJ, Grossman M, et al. Stages of pTDP-43 pathology in amyotrophic lateral sclerosis. Ann Neurol 2013;74:20-38.

[11] Woo CW, Wager TD. Neuroimaging-based biomarker discovery and validation. Pain 2015;156:1379-1381.

[12] Bede P, Hardiman O. Lessons of ALS imaging: pitfalls and future directions - a critical review. Neuroimage Clin 2014;4:436-443.

[13] Chiò A, Traynor BJ. Motor neuron disease in 2014. Biomarkers for ALS-in search of the Promised Land. Nat Rev Neurol 2015;11:72-74.

[14] Verstraete E, Turner MR, Grosskreutz J, Filippi M, Benatar M, attendees of the 4th NiSALS meeting. Mind the gap: the mismatch between clinical and imaging metrics in ALS. Amyotroph Lateral Scler Frontotemporal Degener 2015;16:524-529.

[15] Brooks BR, Bushara K, Khan A, Hershberger J, Wheat JO, Belden D, et al. Functional magnetic resonance imaging (fMRI) clinical studies in ALS - paradigms, problems and promises. Amyotroph Lateral Scler Other Motor Neuron Disord 2000;1 Suppl 2:S23-S32.

[16] Douaud G, Filippini N, Knight S, Talbot K, Turner MR. Integration of structural and functional magnetic resonance imaging in amyotrophic lateral sclerosis. Brain 2011;134:3470-3479.

[17] Turner MR, Agosta F, Bede P, Govind V, Lulé D, Verstraete E. Neuroimaging in amyotrophic lateral sclerosis. Biomark Med 2012;6:319-337.

[18] Trojsi F, Monsurrò MR, Esposito F, Tedeschi G. Widespread structural and functional connectivity changes in amyotrophic lateral sclerosis: insights from advanced neuroimaging research. Neural Plast 2012;2012:473538.

[19] Chiò A, Pagani M, Agosta F, Calvo A, Cistaro A, Filippi M. Neuroimaging in amyotrophic lateral sclerosis: insights into structural and functional changes. Lancet Neurol 2014;13:1228-1240.

[20] Christidi F, Karavasilis E, Rentzos M, Kelekis N, Evdokimidis I, Bede P. Clinical and radiological markers of extra-motor deficits in amyotrophic lateral sclerosis. Front Neurol 2018;9:1005.

[21] Agosta F, Pagani E, Petrolini M, Sormani MP, Caputo D, Perini M, et al. MRI predictors of long-term evolution in amyotrophic lateral sclerosis. Eur J Neurosci 2010;32:1490-1496. 
[22] Agosta F, Chiò A, Cosottini M, De Stefano N, Falini A, Mascalchi M, et al. The present and the future of neuroimaging in amyotrophic lateral sclerosis. AJNR Am J Neuroradiol 2010;31:1769-1777.

[23] Verstraete E, Foerster BR. Neuroimaging as a new diagnostic modality in amyotrophic lateral sclerosis. Neurotherapeutics 2015;12:403-416.

[24] Sach M, Winkler G, Glauche V, Liepert J, Heimbach B, Koch MA, et al. Diffusion tensor MRI of early upper motor neuron involvement in amyotrophic lateral sclerosis. Brain 2004;127:340-350.

[25] Thivard L, Pradat PF, Lehéricy S, Lacomblez L, Dormont D, Chiras J, et al. Diffusion tensor imaging and voxel based morphometry study in amyotrophic lateral sclerosis: relationships with motor disability. J Neurol Neurosurg Psychiatry 2007;78:889-892.

[26] Lulé D, Diekmann V, Müller HP, Kassubek J, Ludolph AC, Birbaumer N. Neuroimaging of multimodal sensory stimulation in amyotrophic lateral sclerosis. J Neurol Neurosurg Psychiatry 2010;81:899-906.

[27] Verstraete E, van den Heuvel MP, Veldink JH, Blanken N, Mandl RC, Hulshoff Pol $\mathrm{HE}$, et al. Motor network degeneration in amyotrophic lateral sclerosis: a structural and functional connectivity study. PLoS One 2010;5:e13664.

[28] Agosta F, Valsasina P, Absinta M, Riva N, Sala S, Prelle A, et al. Sensorimotor functional connectivity changes in amyotrophic lateral sclerosis. Cereb Cortex 2011;21:2291-2298.

[29] Verstraete E, Veldink JH, Mandl RCW, van den Berg LH, van den Heuvel MP. Impaired structural motor connectome in amyotrophic lateral sclerosis. PLoS One 2011;6:e24239.

[30] Canu E, Agosta F, Riva N, Sala S, Prelle A, Caputo D, et al. The topography of brain microstructural damage in amyotrophic lateral sclerosis assessed using diffusion tensor MR imaging. AJNR Am J Neuroradiol 2011;32:1307-1314.

[31] Keil C, Prell T, Peschel T, Hartung V, Dengler R, Grosskreutz J. Longitudinal diffusion tensor imaging in amyotrophic lateral sclerosis. BMC Neurosci 2012;13:141.

[32] Trojsi F, Caiazzo G, Corbo D, Piccirillo G, Cristillo V, Femiano C, et al. Microstructural changes across different clinical milestones of disease in amyotrophic lateral sclerosis. PLoS One 2015;10:e0119045.

[33] Menke RAL, Proudfoot M, Wuu J, Andersen PM, Talbot K, Benatar M, et al. Increased functional connectivity common to symptomatic amyotrophic lateral sclerosis and those at genetic risk. J Neurol Neurosurg Psychiatry 2016;87:580-588. 
[34] Schulthess I, Gorges M, Müller HP, Lulé D, Del Tredici K, Ludolph AC, et al. Functional connectivity changes resemble patterns of pTDP-43 pathology in amyotrophic lateral sclerosis. Sci Rep 2016;6:38391.

[35] Müller HP, Agosta F, Riva N, Spinelli EG, Comi G, Ludolph AC, et al. Fast progressive lower motor neuron disease is an ALS variant: a two-centre tract of interest-based MRI data analysis. Neuroimage Clin 2018;17:145-152.

[36] Menke RAL, Proudfoot M, Talbot K, Turner MR. The two-year progression of structural and functional cerebral MRI in amyotrophic lateral sclerosis. Neuroimage Clin 2018;17:953-961.

[37] Qiu T, Zhang Y, Tang X, Liu X, Wang Y, Zhou C, et al. Precentral degeneration and cerebellar compensation in amyotrophic lateral sclerosis: a multimodal MRI analysis. Hum Brain Mapp 2019;40:3464-3474.

[38] Bharti K, Khan M, Beaulieu C, Graham SJ, Briemberg H, Frayne R, et al. Involvement of the dentate nucleus in the pathophysiology of amyotrophic lateral sclerosis: a multi-center and multi-modal neuroimaging study. Neuroimage Clin 2020;28:102385.

[39] Fox MD, Greicius M. Clinical applications of resting state functional connectivity. Front Syst Neurosci 2010;4:19.

[40] Konrad C, Henningsen H, Bremer J, Mock B, Deppe M, Buchinger C, et al. Pattern of cortical reorganization in amyotrophic lateral sclerosis: a functional magnetic resonance imaging study. Exp Brain Res 2002;143:51-56.

[41] Schoenfeld MA, Tempelmann C, Gaul C, Kühnel GR, Düzel E, Hopf JM, et al. Functional motor compensation in amyotrophic lateral sclerosis. J Neurol 2005;252:944952.

[42] Konrad C, Jansen A, Henningsen H, Sommer J, Turski PA, Brooks BR, et al. Subcortical reorganization in amyotrophic lateral sclerosis. Exp Brain Res 2006;172:361-369.

[43] Tessitore A, Esposito F, Monsurrò MR, Graziano S, Panza D, Russo A, et al. Subcortical motor plasticity in patients with sporadic ALS: an fMRI study. Brain Res Bull 2006;69:489-494.

[44] Stanton BR, Williams VC, Leigh PN, Williams SCR, Blain CRV, Jarosz JM, et al. Altered cortical activation during a motor task in ALS. Evidence for involvement of central pathways. J Neurol 2007;254:1260-1267.

[45] Lulé D, Diekmann V, Kassubek J, Kurt A, Birbaumer N, Ludolph AC, et al. Cortical plasticity in amyotrophic lateral sclerosis: motor imagery and function. Neurorehabil Neural Repair 2007;21:518-526. 
[46] Mohammadi B, Kollewe K, Samii A, Dengler R, Münte TF. Functional neuroimaging at different disease stages reveals distinct phases of neuroplastic changes in amyotrophic lateral sclerosis. Hum Brain Mapp 2011;32:750-758.

[47] Goldstein LH, Newsom-Davis IC, Bryant V, Brammer M, Leigh PN, Simmons A. Altered patterns of cortical activation in ALS patients during attention and cognitive response inhibition tasks. J Neurol 2011;258:2186-2198.

[48] Cosottini M, Pesaresi I, Piazza S, Diciotti S, Cecchi P, Fabbri S, et al. Structural and functional evaluation of cortical motor areas in amyotrophic lateral sclerosis. Exp Neurol 2012;234:169-180.

[49] Passamonti L, Fera F, Tessitore A, Russo A, Cerasa A, Gioia CM, et al. Dysfunctions within limbic-motor networks in amyotrophic lateral sclerosis. Neurobiol Aging 2013;34:2499-2509.

[50] Witiuk K, Fernandez-Ruiz J, McKee R, Alahyane N, Coe BC, Melanson M, et al. Cognitive deterioration and functional compensation in ALS measured with fMRI using an inhibitory task. J Neurosci 2014;34:14260-14271.

[51] Mohammadi B, Kollewe K, Cole DM, Fellbrich A, Heldmann M, Samii A, et al. Amyotrophic lateral sclerosis affects cortical and subcortical activity underlying motor inhibition and action monitoring. Hum Brain Mapp 2015;36:2878-2889.

[52] Li H, Chen Y, Li Y, Yin B, Tang W, Yu X, et al. Altered cortical activation during action observation in amyotrophic lateral sclerosis patients: a parametric functional MRI study. Eur Radiol 2015;25:2584-2592.

[53] Jelsone-Swain L, Persad C, Burkard D, Welsh RC. Action processing and mirror neuron function in patients with amyotrophic lateral sclerosis: an fMRI study. PLoS One 2015;10:e0119862.

[54] Mohammadi B, Kollewe K, Samii A, Krampfl K, Dengler R, Münte TF. Changes of resting state brain networks in amyotrophic lateral sclerosis. Exp Neurol 2009;217:147-153.

[55] Jelsone-Swain LM, Fling BW, Seidler RD, Hovatter R, Gruis K, Welsh RC. Reduced interhemispheric functional connectivity in the motor cortex during rest in limb-onset amyotrophic lateral sclerosis. Front Syst Neurosci 2010;4:158.

[56] Chenji S, Jha S, Lee D, Brown M, Seres P, Mah D, et al. Investigating default mode and sensorimotor network connectivity in amyotrophic lateral sclerosis. PLoS One 2016;11:e0157443. 
[57] Xu J, Li H, Li C, Yao JC, Hu J, Wang J, et al. Abnormal cortical-basal ganglia network in amyotrophic lateral sclerosis: a voxel-wise network efficiency analysis. Behav Brain Res 2017;333:123-128.

[58] Fang X, Zhang Y, Wang Y, Zhang Y, Hu J, Wang J, et al. Disrupted effective connectivity of the sensorimotor network in amyotrophic lateral sclerosis. J Neurol 2016;263:508-516.

[59] Proudfoot M, Bede P, Turner MR. Imaging cerebral activity in amyotrophic lateral sclerosis. Front Neurol 2019;9:1148.

[60] Van Dijk KRA, Hedden T, Venkataraman A, Evans KC, Lazar SW, Buckner RL. Intrinsic functional connectivity as a tool for human connectomics: theory, properties, and optimization. J Neurophysiol 2010;103:297-321.

[61] Verstraete E, Biessels GJ, van Den Heuvel MP, Visser F, Luijten PR, van Den Berg LH. No evidence of microbleeds in ALS patients at 7 Tesla MRI. Amyotroph Lateral Scler 2010;11:555-557.

[62] Kwan JY, Jeong SY, Van Gelderen P, Deng HX, Quezado MM, Danielian LE, et al. Iron accumulation in deep cortical layers accounts for MRI signal abnormalities in ALS: correlating 7 Tesla MRI and pathology. PLoS One 2012;7:e35241.

[63] Cosottini M, Donatelli G, Costagli M, Caldarazzo Ienco E, Frosini D, Pesaresi I, et al. High-resolution 7T MR imaging of the motor cortex in amyotrophic lateral sclerosis. AJNR Am J Neuroradiol 2016;37:455-461.

[64] Costagli M, Donatelli G, Biagi L, Caldarazzo Ienco E, Siciliano G, Tosetti M, et al. Magnetic susceptibility in the deep layers of the primary motor cortex in amyotrophic lateral sclerosis. Neuroimage Clin 2016;12:965-969.

[65] Verstraete E, Polders DL, Mandl RCW, Van Den Heuvel MP, Veldink JH, Luijten P, et al. Multimodal tract-based analysis in ALS patients at 7T: a specific white matter profile? Amyotroph Lateral Scler Frontotemporal Degener 2014;15:84-92.

[66] Atassi N, Xu M, Triantafyllou C, Keil B, Lawson R, Cernasov P, et al. Ultra high-field (7tesla) magnetic resonance spectroscopy in amyotrophic lateral sclerosis. PLoS One 2017;12:e0177680.

[67] Cheong I, Marjańska M, Deelchand DK, Eberly LE, Walk D, Öz G. Ultra-high field proton MR spectroscopy in early-stage amyotrophic lateral sclerosis. Neurochem Res 2017;42:1833-1844.

[68] Cohen-Adad J, Zhao W, Keil B, Ratai EM, Triantafyllou C, Lawson R, et al. 7-T MRI of the spinal cord can detect lateral corticospinal tract abnormality in amyotrophic lateral sclerosis. Muscle Nerve 2013;47:760-762. 
[69] Ladd ME, Bachert P, Meyerspeer M, Moser E, Nagel AM, Norris DG, et al. Pros and cons of ultra-high-field MRI/MRS for human application. Prog Nucl Magn Reson Spectrosc 2018;109:1-50.

[70] Obusez EC, Lowe M, Oh SH, Wang I, Bullen J, Ruggieri P, et al. 7T MR of intracranial pathology: preliminary observations and comparisons to $3 \mathrm{~T}$ and $1.5 \mathrm{~T}$. Neuroimage 2018;168:459-476.

[71] Donatelli G, Ceravolo R, Frosini D, Tosetti M, Bonuccelli U, Cosottini M. Present and future of ultra-high field MRI in neurodegenerative disorders. Curr Neurol Neurosci Rep 2018;18:31.

[72] Barry RL, Vannesjo SJ, By S, Gore JC, Smith SA. Spinal cord MRI at 7T. Neuroimage 2018;168:437-451.

[73] Cedarbaum JM, Stambler N, Malta E, Fuller C, Hilt D, Thurmond B, et al. The ALSFRS-R: a revised ALS functional rating scale that incorporates assessments of respiratory function. J Neurol Sci 1999;169:13-21.

[74] Keil B, Triantafyllou C, Hamm M, Wald LL. Design optimization of a 32-channel head coil at 7T. Proc Int Soc Magn Reson Med 2010;18:1493.

[75] Whitfield-Gabrieli S, Nieto-Castanon A. Conn: a functional connectivity toolbox for correlated and anticorrelated brain networks. Brain Connect 2012;2:125-141.

[76] Evans AC, Collins DL, Mills SR, Brown ED, Kelly RL, Peters TM. 3D statistical neuroanatomical models from 305 MRI volumes. Proc IEEE Nucl Sci Symp Med Imaging Conf 1993;p. 1813-1817.

[77] Mikl M, Mareček R, Hluštík P, Pavlicová M, Drastich A, Chlebus P, et al. Effects of spatial smoothing on fMRI group inferences. Magn Reson Imaging 2008;26:490-503.

[78] Behzadi Y, Restom K, Liau J, Liu TT. A component based noise correction method (CompCor) for BOLD and perfusion based fMRI. Neuroimage 2007;37:90-101.

[79] Diedrichsen J. A spatially unbiased atlas template of the human cerebellum. Neuroimage 2006;33:127-138.

[80] Diedrichsen J, Balsters JH, Flavell J, Cussans E, Ramnani N. A probabilistic MR atlas of the human cerebellum. Neuroimage 2009;46:39-46.

[81] Diedrichsen J, Maderwald S, Küper M, Thürling M, Rabe K, Gizewski ER, et al. Imaging the deep cerebellar nuclei: a probabilistic atlas and normalization procedure. Neuroimage 2011;54:1786-1794. 
[82] Diedrichsen J, Zotow E. Surface-based display of volume-averaged cerebellar imaging data. PLoS One 2015;10:e0133402.

[83] Power JD, Barnes KA, Snyder AZ, Schlaggar BL, Petersen SE. Spurious but systematic correlations in functional connectivity MRI networks arise from subject motion. Neuroimage 2012;59:2142-2154.

[84] Dockès J, Poldrack RA, Primet R, Gözükan H, Yarkoni T, Suchanek F, et al. NeuroQuery, comprehensive meta-analysis of human brain mapping. Elife 2020;9:e53385.

[85] Mackenzie IRA, Frick P, Neumann M. The neuropathology associated with repeat expansions in the C9ORF72 gene. Acta Neuropathol 2014;127:347-357.

[86] Bede P, Elamin M, Byrne S, McLaughlin RL, Kenna K, Vajda A, et al. Patterns of cerebral and cerebellar white matter degeneration in ALS. J Neurol Neurosurg Psychiatry 2015;86:468-470.

[87] Wang MD, Gomes J, Cashman NR, Little J, Krewski D. Intermediate CAG repeat expansion in the ATXN2 gene is a unique genetic risk factor for ALS-a systematic review and meta-analysis of observational studies. PLoS One 2014;9:e105534.

[88] Wilke C, Baets J, De Bleecker JL, Deconinck T, Biskup S, Hayer SN, et al. Beyond ALS and FTD: the phenotypic spectrum of TBK1 mutations includes PSP-like and cerebellar phenotypes. Neurobiol Aging 2018;62:244.e9-244.e13.

[89] Stoodley CJ, Schmahmann JD. Functional topography of the human cerebellum. Handb Clin Neurol 2018;154:59-70.

[90] Nitschke MF, Binkofski F, Buccino G, Posse S, Erdmann C, Kömpf D, et al. Activation of cerebellar hemispheres in spatial memorization of saccadic eye movements: an fMRI study. Hum Brain Mapp 2004;22:155-164.

[91] Konen CS, Kleiser R, Seitz RJ, Bremmer F. An fMRI study of optokinetic nystagmus and smooth-pursuit eye movements in humans. Exp Brain Res 2005;165:203-216.

[92] Schraa-Tam CKL, van Broekhoven P, van der Geest JN, Frens MA, Smits M, van der Lugt A. Cortical and cerebellar activation induced by reflexive and voluntary saccades. Exp Brain Res 2009;192:175-187.

[93] Urban PP, Marx J, Hunsche S, Gawehn J, Vucurevic G, Wicht S, et al. Cerebellar speech representation: lesion topography in dysarthria as derived from cerebellar ischemia and functional magnetic resonance imaging. Arch Neurol 2003;60:965-972.

[94] Dresel C, Castrop F, Haslinger B, Wohlschlaeger AM, Hennenlotter A, CeballosBaumann AO. The functional neuroanatomy of coordinated orofacial movements: sparse sampling fMRI of whistling. Neuroimage 2005;28:588-597. 
[95] Imamizu H, Kawato M. Brain mechanisms for predictive control by switching internal models: implications for higher-order cognitive functions. Psychol Res 2009;73:527544 .

[96] Schlerf JE, Verstynen TD, Ivry RB, Spencer RMC. Evidence of a novel somatopic map in the human neocerebellum during complex actions. J Neurophysiol 2010;103:33303336 .

[97] Habas C, Kamdar N, Nguyen D, Prater K, Beckmann CF, Menon V, et al. Distinct cerebellar contributions to intrinsic connectivity networks. J Neurosci 2009;29:85868594 .

[98] Krienen FM, Buckner RL. Segregated fronto-cerebellar circuits revealed by intrinsic functional connectivity. Cereb Cortex 2009;19:2485-2497.

[99] O’Reilly JX, Beckmann CF, Tomassini V, Ramnani N, Johansen-Berg H. Distinct and overlapping functional zones in the cerebellum defined by resting state functional connectivity. Cereb Cortex 2010;20:953-965.

[100] Abidi M, de Marco G, Couillandre A, Feron M, Mseddi E, Termoz N, et al. Adaptive functional reorganization in amyotrophic lateral sclerosis: coexisting degenerative and compensatory changes. Eur J Neurol 2020;27:121-128.

[101] Floeter MK, Katipally R, Kim MP, Schanz O, Stephen M, Danielian L, et al. Impaired corticopontocerebellar tracts underlie pseudobulbar affect in motor neuron disorders. Neurology 2014;83:620-627.

[102] Bede P, Hardiman O. Longitudinal structural changes in ALS: a three time-point imaging study of white and gray matter degeneration. Amyotroph Lateral Scler Frontotemporal Degener 2018;19:232-241.

[103] Tu S, Menke RAL, Talbot K, Kiernan MC, Turner MR. Cerebellar tract alterations in PLS and ALS. Amyotroph Lateral Scler Frontotemporal Degener 2019;20:281-284.

[104] Prell T, Grosskreutz J. The involvement of the cerebellum in amyotrophic lateral sclerosis. Amyotroph Lateral Scler Frontotemporal Degener 2013;14:507-515.

[105] Han J, Ma L. Functional magnetic resonance imaging study of the brain in patients with amyotrophic lateral sclerosis. Chin Med Sci J 2006;21:228-233.

[106] Zhou F, Gong H, Li F, Zhuang Y, Zang Y, Xu R, et al. Altered motor network functional connectivity in amyotrophic lateral sclerosis: a resting-state functional magnetic resonance imaging study. Neuroreport 2013;24:657-662.

[107] Cole DM, Smith SM, Beckmann CF. Advances and pitfalls in the analysis and interpretation of resting-state FMRI data. Front Syst Neurosci 2010;4:8. 
[108] Beisteiner R, Robinson S, Wurnig M, Hilbert M, Merksa K, Rath J, et al. Clinical fMRI: evidence for a 7T benefit over 3T. Neuroimage 2011;57:1015-1021.

[109] Menon RS, Ogawa S, Tank DW, Uğurbil K. 4 Tesla gradient recalled echo characteristics of photic stimulation-induced signal changes in the human primary visual cortex. Magn Reson Med 1993;30:380-386.

[110] Gati JS, Menon RS, Uğurbil K, Rutt BK. Experimental determination of the BOLD field strength dependence in vessels and tissue. Magn Reson Med 1997;38:296-302.

[111] Krüger G, Kastrup A, Glover GH. Neuroimaging at $1.5 \mathrm{~T}$ and $3.0 \mathrm{~T}$ : comparison of oxygenation-sensitive magnetic resonance imaging. Magn Reson Med 2001;45:595604 .

[112] Triantafyllou C, Hoge RD, Krueger G, Wiggins CJ, Potthast A, Wiggins GC, et al. Comparison of physiological noise at $1.5 \mathrm{~T}, 3 \mathrm{~T}$ and $7 \mathrm{~T}$ and optimization of fMRI acquisition parameters. Neuroimage 2005;26:243-250.

[113] Triantafyllou C, Hoge RD, Wald LL. Effect of spatial smoothing on physiological noise in high-resolution fMRI. Neuroimage 2006;32:551-557.

[114] Hutchison RM, Womelsdorf T, Allen EA, Bandettini PA, Calhoun VD, Corbetta $\mathrm{M}$, et al. Dynamic functional connectivity: promise, issues, and interpretations. Neuroimage 2013;80:360-378.

[115] Chen JE, Glover GH. BOLD fractional contribution to resting-state functional connectivity above $0.1 \mathrm{~Hz}$. Neuroimage 2015;107:207-218.

[116] Gohel SR, Biswal BB. Functional integration between brain regions at rest occurs in multiple-frequency bands. Brain Connect 2015;5:23-34.

[117] Chen JE, Polimeni JR, Bollmann S, Glover GH. On the analysis of rapidly sampled fMRI data. Neuroimage 2019;188:807-820.

[118] Birn RM, Molloy EK, Patriat R, Parker T, Meier TB, Kirk GR, et al. The effect of scan length on the reliability of resting-state fMRI connectivity estimates. Neuroimage 2013;83:550-558.

[119] Kew JJM, Brooks DJ, Passingham RE, Rothwell JC, Frackowiak RSJ, Leigh PN. Cortical function in progressive lower motor neuron disorders and amyotrophic lateral sclerosis: a comparative PET study. Neurology 1994;44:1101-1110.

[120] Frazier JA, Chiu S, Breeze JL, Makris N, Lange N, Kennedy DN, et al. Structural brain magnetic resonance imaging of limbic and thalamic volumes in pediatric bipolar disorder. Am J Psychiatry 2005;162:1256-1265. 
[121] Moeller S, Yacoub E, Olman CA, Auerbach E, Strupp J, Harel N, et al. Multiband multislice GE-EPI at 7 Tesla, with 16-fold acceleration using partial parallel imaging with application to high spatial and temporal whole-brain FMRI. Magn Reson Med 2010;63:1144-1153.

[122] Beeldman E, Govaarts R, de Visser M, van Es MA, Pijnenburg YAL, Schmand BA, et al. Screening for cognition in amyotrophic lateral sclerosis: test characteristics of a new screen. J Neurol 2021;in press.

[123] Mormina E, Petracca M, Bommarito G, Piaggio N, Cocozza S, Inglese M. Cerebellum and neurodegenerative diseases: beyond conventional magnetic resonance imaging. World J Radiol 2017;9:371-388.

[124] Gellersen HM, Guo CC, O'Callaghan C, Tan RH, Sami S, Hornberger M. Cerebellar atrophy in neurodegeneration - a meta-analysis. J Neurol Neurosurg Psychiatry 2017;88:780-788. 